\title{
Conditional Replication Adenovirus Sensitizes A549 Cancer Cell to Cisplatin
}

\author{
Yanan Liu, Yinghui Huang \\ China-Japan Union Hospital of Jilin university, Jilin, China \\ Email: dl0009@sina.com, yhuang@jlu.edu.cn
}

Received 2012

\begin{abstract}
Objective: The aim of the study was to improve the therapeutic effect for lung cancer using a synergetic strategy of adenovirus-based gene therapy combined with chemotherapy. Methods: A conditional replication adenovirus(CRAd) was employed to treat the A549 lung cancer cells and cisplatin-resisted A549(A549-DDP) cells. In vitro MTS / PMS assay were used to evaluate the cell viability. PCR were used to detect expression of Coxsackie receptor (CAR) and Multidrug resistance(MDR) gene. The in vivo anti-tumor effect of CRAd and cisplatin was evaluated using a subcutaneous mouse model. Results: The CRAd sensitizes A549 cancer cells to cisplatin. The mechanism of enhanced cell growth inhibition is associated with the increased CAR and MDR expression. Conclusion: Our approach is better than the conventional gene therapy and chemotherapy strategy.
\end{abstract}

Keywords: Cisplatin; Adenovirus; Lung Cancer

\section{Introduction}

Lung cancer is a common malignancy [1], the majority of patients lost the chance of surgery when found, chemotherapy play a very important role in the clinical treatment of lung cancer and prolong survival in patients [2,3], but resistance of chemotherapy drug has hindered its development [4]. Overexpression of the MDR gene is one of the mechanisms leads to tumor cell multidrug resistance [5], while adenovirus cancer gene therapy has rapidly developed in natural medicine, conditionally replicative adenovirus (CRAd) is able to selectively copy in tumor cells and spread to more tumor cells, CRAd not only has a stronger tumor scavenging, but also to reduce the liver toxic effects of less dosage. This experiment combined CRAd with the chemotherapy drug cisplatin, explore the effect and mechanisms of A549 and A549-DDP.

\section{Methods}

\subsection{MTS/PMS Assay}

A549, A549-DDP cells were seeded in 24-well plates, at a density of $1 \times 10^{5}$ per culture well,after 24 hours, each well (a) treated three hours with different concentrations of cisplatin 0.25ug/ml, $1 \mathrm{ug} / \mathrm{ml}, 4 \mathrm{ug} / \mathrm{ml}, 16 \mathrm{ug} / \mathrm{ml}, 64 \mathrm{ug} / \mathrm{ml}$; (b) treated with different concentrations of CRAd virus 100MOI, 200MOI, 500MOI, 1000MOI, 2000MOI; (c) the treatment group each hole by adding 100MOI CRAd for four hours, and then different concentrations of cisplatin for three hours; (d) treated with each hole by adding different concentrations of cisplatin for three hours, then add 100MOI CRAd for four hours; different treated cells transferred to 96 -well plate, three wells, $5 \times 10^{3}$ cells per well, and observed for 4 days to add the MTS / PMS reagents,the absorbance at $490 \mathrm{~nm}$ was detected.

\subsection{Semiquantitative Reverse Transcription PCR}

A549 and A549- DDP cells were collected , total RNA was extracted, each sample was converted to complementary cDNA , primer sequence is below:

GAPDH: S: 5'GATTGTTGCCATCAACGACC3 ' AS:

5 'GTGCAGGATGCATTGCTGAC 3' 371bp CAR:

S: 5 'CCACCTCCAAAGAGCCGTAC 3' AS:

5 'ATCACAGGAATCGCACCC 3' 218bp MDR:

S: 5 'TCGTAGGAGTGTCCGTGGAT 3' AS:

5 'CATTGGCGAGCCTGGTAG 3' 417bp

\subsection{Tumor Model}

BALB/C nude mice, female (6-8 weeks old) were acquired from the Chinese Academy of Sciences, A549, A549- DDP cells $\left(4 \times 10^{6}\right)$ inoculated subcutaneous into the right flank of mice, Tumors were visible on the 15 th day.

\subsection{In Vivo Tumor Inhibiton Assay}

BALB/C nude mice, female (6-8 weeks old). A549 (4 x 10 ${ }^{6}$ ), A549-DDP $\left(4 \times 10^{6}\right)$ cells were inoculated subcutaneous into the right flank of mice ,tumor bearing mice( $n=6$ per group) were divided into three groups.Mice in each group were treated as follow: (a) cisplatin treatment group (b) CRAd treatment group (c) cisplatin combined CRAd treatment group (first give cislatin then give CRAd); Ad-luc virus transfected to each treatment group, a certain period of time to observe the effect by vivo imaging.

\section{Results}

1) After treated with different concentrations of cisplatin, cisplatin can inhibit the proliferation of A549 cells, but the A549-DDP cell proliferation inhibition was not obvious.

2) After treated with different concentrations of CRAd virus, 
CRAd virus can inhibit the proliferation of A549 and A549DDP cells, the inhibitory effect of the CRAd virus on A549DDP cell line is more obvious.

3) Treated A549 and A549- DDP cells by combined with cisplatin and CRAd virus, we found that the inhibition of cell treated by the method mentioned at MTS/PMS assay group (d) is more obvious than group (c) on A549-DDP cell line

4) PCR results showed that the expression of CAR in A549-DDP cells increased, expression of MDR in A549-DDP cells also increased .

5) Tumor in vivo experimental results show that the A549 and A549-DDP cell line can form tumor. In vivo inhibition experiments showed that cisplatin combined with CRAd virus group treatment is the most valid on tumor growth (data not shown).

\section{Discussion}

Our experiments found that cisplatin combined virus is more effects than the separate application of cisplatin, or separate

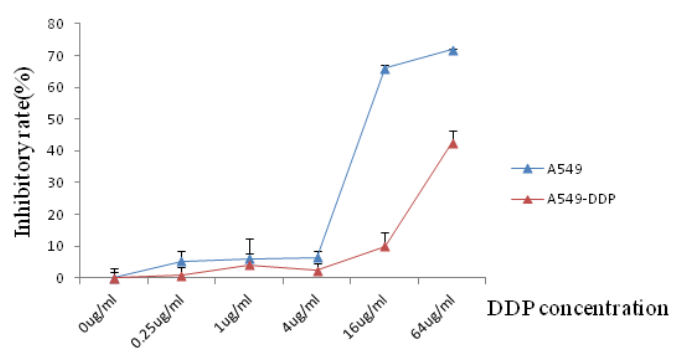

Figure 1. Inhibitory effects evaluated with MTS/PMS assay for the A549 and A549-DDP cells treated with different concentration of DDP.

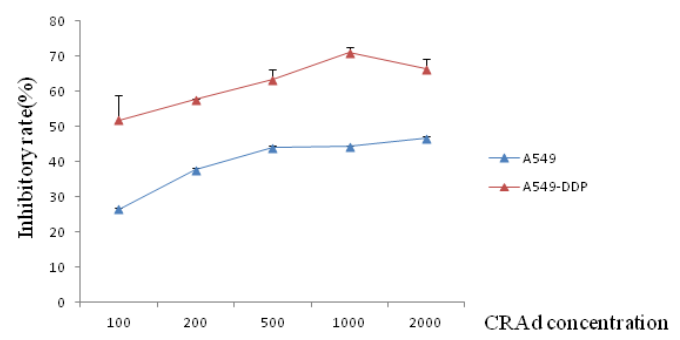

Figure 2. Inhibitory effects evaluated with MTS/PMS assay for the A549 and A549-DDP cells treated with different concentration of CRAd.

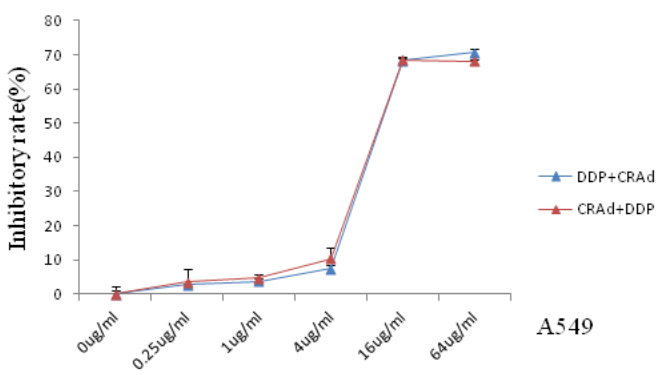

Figure 3. Inhibitory effects evaluated with MTS/PMS assay for the A549 cells treated with DDP and CRAd.

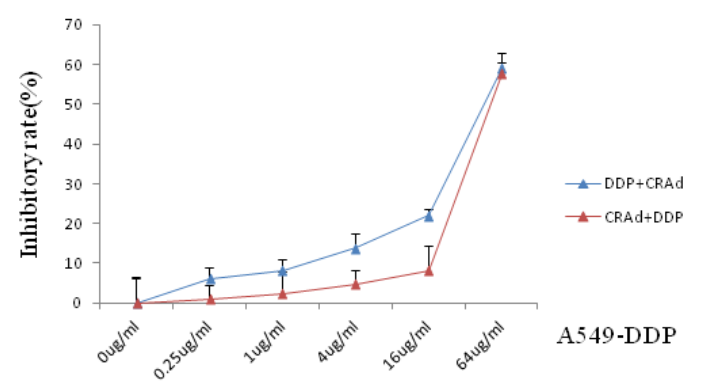

Figure 4. Inhibitory effects evaluated with MTS/PMS assay for the A549-DDP cells treated with DDP and CRAd.

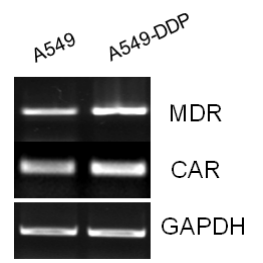

Figure 5. Gene expression of MDR and CAR were examined with PCR.

application of virus on tumor cells, its mechanism may be related to the adenovirus receptor CAR expression upregulation on the A549-DDP resistant cell lines. The key aspects of adenoviral transduction of adenovirus is combined with the cell surface coxsackie adenovirus receptor (CAR), our experimental results show that the A549-DDP cell surface of CAR receptor expression enhanced, making the virus is more easily infected tumor cells, that is why the effect of combined treatment better than simple treatment. There are another possible mechanism that high expression of MDR gene on A549-DDP cell affects the chemotherapy, combination therapy can enhance the therapeutic effect of chemotherapy . Tumor in vivo experimental results show that both A549 and A549-DDP cell can form tumor. The in vivo inhibition of experimental results show that the cisplatin combined with CRAd virus group is not only can inhibit the A549 cell lines, but also can play a good role in inhibition of tumor growth resistant cell line A549-DDP. Its mechanism may still be raised Coxsackie and adenovirus receptor of tumor cells and affect the expression of MDR.

\section{REFERENCES}

[1] Siegel R, Naishadham D, Jemal A. Cancer statistics, 2012. CA Cancer J Clin.2012:62 (1) :10-29.

[2] Socinski MA, Bogart JA. Limited-stage small-cell lung cancer: the current status of combined-modality therapy. J Clin Oncol 2007,25: 4137-4145.

[3] Wan L,Hyo-Im C,Mi-Jin K, et a.l Depletion ofmitochondrial DNA up-regulates the expression of MDR1 gene via an increase in mRNA stability.Exp.Mo.lMed, 2008, 40(1): 109-117.

[4] Paredes Lario A, Blanco Garcia C et al.Expression of proteins associated with multidrug resistance and resistance to chemotherapy in lung cancer[J].Arch Bronconeumol, 2007;43(9):479 484.

[5] Pujol JL,Simony J,Gautier V,et al.Immuno-histochemical study of p-glycoprotein distribution in lung [J].Cancer,1993,10:1. 